\title{
Consensus scoring systems for nonalcoholic fatty liver disease: an unmet clinical need
}

\author{
Yu-Jie Zhou ${ }^{1,2}$, Vincent Wai-Sun Wong ${ }^{3,4}$, Ming-Hua Zheng ${ }^{1,5,6}$ \\ ${ }^{1}$ NAFLD Research Center, Department of Hepatology, the First Affiliated Hospital of Wenzhou Medical University, Wenzhou, China; ${ }^{2}$ Division \\ of Gastroenterology and Hepatology, Key Laboratory of Gastroenterology and Hepatology, Ministry of Health, Shanghai Institute of Digestive \\ Disease, Renji Hospital, School of Medicine, Shanghai Jiao Tong University, Shanghai, China; ${ }^{3}$ Department of Medicine and Therapeutics, The \\ Chinese University of Hong Kong, Hong Kong, China; ${ }^{4}$ State Key Laboratory of Digestive Disease, The Chinese University of Hong Kong, \\ Hong Kong, China; ${ }^{5}$ Institute of Hepatology, Wenzhou Medical University, Wenzhou, China; ${ }^{6}$ Key Laboratory of Diagnosis and Treatment for the \\ Development of Chronic Liver Disease in Zhejiang Province, Wenzhou, China \\ Correspondence to: Ming-Hua Zheng, MD, PhD. NAFLD Research Center, Department of Hepatology, the First Affiliated Hospital of Wenzhou \\ Medical University, No. 2 Fuxue Lane, Wenzhou 325000, China. Email: zhengmh@wmu.edu.cn; Vincent Wai-Sun Wong, MD. Department of \\ Medicine and Therapeutics, 9/F Prince of Wales Hospital, 30-32 Ngan Shing Street, Shatin, Hong Kong, China. Email: wongv@cuhk.edu.hk.
}

Submitted Feb 22, 2021. Accepted for publication Apr 19, 2021.

doi: $10.21037 / \mathrm{hbsn}-21-80$

View this article at: http://dx.doi.org/10.21037/hbsn-21-80

Noninvasive diagnosis of nonalcoholic fatty liver disease (NAFLD) has received increasing attention $(1,2)$. Nowadays, when we search the item 'scoring system and fatty liver' in PubMed, we can see a sharp increase in the number of related papers in the past few years. In fact, an era of 'information explosion' of noninvasive tests (NITs) for NAFLD is coming. For one thing, these newly identified biomarkers or noninvasive panels allow clinicians to detect nonalcoholic steatohepatitis (NASH) and liver fibrosis without the need for liver biopsy; for another, there are too many NITs for clinicians to choose from. Clinicians may be at a loss as to which NIT to use in different clinical contexts.

NITs for NAFLD can be classified according to the target of interest, namely steatosis, NASH, significant/ advanced fibrosis, and more recently the so-called 'fibrotic NASH' $(3,4)$, based on either serum-based tests or imaging (such as ultrasound scan, vibration-controlled transient elastography or magnetic resonance elastography) $(5,6)$. NITs based on widely-available imaging techniques, such as Hamaguchi score and the ultrasonographic fatty liver indicator (US-FLI), are derived from ultrasonographic findings $(7,8)$. Serum-based NITs incorporate readily accessible clinical parameters or novel biomarkers, both of which have merits and demerits. NITs based on routine clinical parameters (such as the fatty liver index, NAFLD fibrosis score, Fibrosis-4 index, Hepamet fibrosis score (9), and aspartate aminotransferase-to-platelet ratio index) are simple and easy to use but have modest accuracies. They can be easily confounded by several factors like liver enzymes and age (10); also, NITs with dual-cutoffs often leave a significant proportion of patients in the gray zone. Some of these scores were originally derived in patients with other liver diseases (11). NITs based on novel biomarkers, on the other hand, have more reproducible results with definite biological meanings, particularly those novel markers for liver fibrosis; however, these biomarkers may be expensive and not widely available, and we need to figure out how to integrate some of these novel indicators into clinical practice. The reliability and feasibility of some 'omics' markers of gene loci and metabolites remain to be validated in the future.

NAFLD biomarkers and surrogate scores, can target the following domains: (I) diagnostic markers reflecting the stage of fibrosis or NASH; (II) prognostic markers, stratifying the fibrosis progression risk; (III) monitoring biomarkers that may be used to track the disease progression and/or the treatment response $(12,13)$. In the last two decades, some progress has been made in detection of new biomarkers and subsequently new NITs in hepatology. However, for many of them further independent validations are need. In reality, only a few 


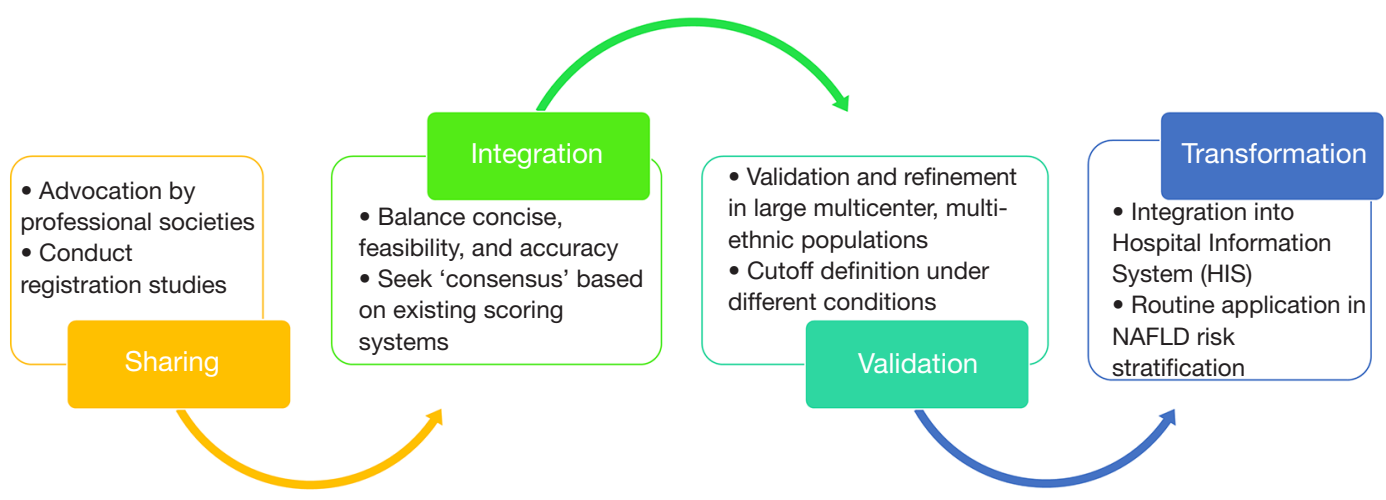

Figure 1 A diagram to illustrate procedures to generate 'consensus scoring systems' for clinical management of NAFLD. NAFLD, nonalcoholic fatty liver disease.

NITs have been well validated in more than three cohorts. Many scoring systems work well in the original reports but have much lower accuracies when tested in NAFLD cohorts of different clinical and ethnic compositions, limiting their value for wider clinical application. In some way, these endless newly-published panels without sufficient clinical validation can cause the reader to feel 'esthetic fatigue'. As a saying goes: 'less is more', it is high time that we streamline these existing NITs to facilitate clinical usage (Figure 1).

Firstly, professional societies [European Association for the Study of the Liver (EASL), American Association for the Study of Liver Diseases (AASLD), and Asian Pacific Association for the Study of the Liver (APASL)] can advocate for data sharing to integrate resources (raw data from randomized clinical trials (RCTs), prospectively collected clinical cohorts, etc.), in order to conduct registration studies and establish a high-quality global database based on pathological diagnosis [such as Liver Investigation: Testing Marker Utility in Steatohepatitis (LITMUS) (14)]. Secondly, by integration of existing resources, we can compare and identify the common elements in the existing scoring systems, and develop a more accurate, more pragmatic 'consensus scoring system', taking into account both clinical availability and novelty/accuracy. Thirdly, the 'consensus scoring system' needs to be prospectively validated and refined in large multicenter, multi-ethnic populations with diverse background (e.g., ongoing RCT) and determine optimal cutoff values under different contexts. Lastly, the 'consensus scoring system' can finally be transformed into daily clinical application in NAFLD risk stratification and prognosis evaluation (such as integration into the hospital information system), in order to better guide the clinical diagnosis and treatment of NAFLD.

\section{Acknowledgments}

Funding: This work was supported by grants from the National Natural Science Foundation of China (82070588), High Level Creative Talents from Department of Public Health in Zhejiang Province (S2032102600032) and Project of New Century 551 Talent Nurturing in Wenzhou.

\section{Footnote}

Provenance and Peer Review: This article was commissioned by the editorial office of Hepatobiliary Surgery and Nutrition. The article has undergone external peer review.

Conflicts of Interest: All authors have completed the ICMJE uniform disclosure form (available at https://hbsn. amegroups.com/article/view/10.21037/hbsn-21-80/coif). VWSW served as a consultant and speaker for Echosens. The other authors have no conflicts of interest to declare.

Ethical Statement: The authors are accountable for all aspects of the work in ensuring that questions related to the accuracy or integrity of any part of the work are appropriately investigated and resolved.

Open Access Statement: This is an Open Access article distributed in accordance with the Creative Commons 
Attribution-NonCommercial-NoDerivs 4.0 International License (CC BY-NC-ND 4.0), which permits the noncommercial replication and distribution of the article with the strict proviso that no changes or edits are made and the original work is properly cited (including links to both the formal publication through the relevant DOI and the license). See: https://creativecommons.org/licenses/by-nc-nd/4.0/.

\section{References}

1. Younossi ZM, Noureddin M, Bernstein D, et al. Role of Noninvasive Tests in Clinical Gastroenterology Practices to Identify Patients With Nonalcoholic Steatohepatitis at High Risk of Adverse Outcomes: Expert Panel Recommendations. Am J Gastroenterol 2021;116:254-62.

2. Zhou YJ, Zheng KI, Targher G, et al. Non-invasive diagnosis of non-alcoholic steatohepatitis and liver fibrosis. Lancet Gastroenterol Hepatol 2021;6:9-10.

3. Canivet C, Moal V, Zuberbuhler F, et al. MACK-3: an accurate blood test for the diagnosis of fibrotic NASH and a candidate biomarker to monitor disease progression. J Hepatol 2020;73:S401-652.

4. Boursier J, Anty R, Vonghia L, et al. Screening for therapeutic trials and treatment indication in clinical practice: MACK-3, a new blood test for the diagnosis of fibrotic NASH. Aliment Pharmacol Ther 2018;47:1387-96.

5. Wong VW, Adams LA, de Lédinghen V, et al. Noninvasive biomarkers in NAFLD and NASH - current progress and future promise. Nat Rev Gastroenterol Hepatol 2018;15:461-78.

6. Rios RS, Zheng KI, Targher G, et al. Non-invasive fibrosis assessment in non-alcoholic fatty liver disease. Chin Med J (Engl) 2020;133:2743-5.

7. Hamaguchi M, Kojima T, Itoh $Y$, et al. The severity of ultrasonographic findings in nonalcoholic fatty liver disease reflects the metabolic syndrome and visceral fat accumulation. Am J Gastroenterol 2007;102:2708-15.

8. Ballestri S, Nascimbeni F, Baldelli E, et al. Ultrasonographic fatty liver indicator detects mild steatosis and correlates with metabolic/histological parameters in various liver diseases. Metabolism 2017;72:57-65.

9. Ampuero J, Pais R, Aller R, et al. Development and Validation of Hepamet Fibrosis Scoring System-A Simple, Noninvasive Test to Identify Patients With Nonalcoholic Fatty Liver Disease With Advanced Fibrosis. Clin Gastroenterol Hepatol 2020;18:216-25.e5.

10. Wai JW, Fu C, Wong VW. Confounding factors of non-invasive tests for nonalcoholic fatty liver disease. J Gastroenterol 2020;55:731-41.

11. Sterling RK, Lissen E, Clumeck N, et al. Development of a simple noninvasive index to predict significant fibrosis in patients with HIV/HCV coinfection. Hepatology 2006;43:1317-25.

12. Loomba R, Adams LA. Advances in non-invasive assessment of hepatic fibrosis. Gut 2020;69:1343-52.

13. Klisic A, Abenavoli L, Fagoonee S, et al. Older age and HDL-cholesterol as independent predictors of liver fibrosis assessed by BARD score. Minerva Med 2019;110:191-8.

14. Hardy T, Wonders K, Younes R, et al. The European NAFLD Registry: A real-world longitudinal cohort study of nonalcoholic fatty liver disease. Contemp Clin Trials 2020;98:106175.
Cite this article as: Zhou YJ, Wong VWS, Zheng MH. Consensus scoring systems for nonalcoholic fatty liver disease: an unmet clinical need. HepatoBiliary Surg Nutr 2021;10(3):388390. doi: 10.21037/hbsn-21-80 